\title{
Come together: bioelectric healing-on-a-chip
}

\author{
Tom J. Zajdel ${ }^{a}$, Gawoon Shim ${ }^{a}$, Daniel J. Cohen* ${ }^{a}$
}

\author{
${ }^{a}$ Department of Mechanical \& Aerospace Engineering, Princeton University, Princeton, NJ, \\ United States of America \\ * Corresponding author: danielcohen@princeton.edu
}

\begin{abstract}
There is a growing interest in bioelectric wound treatment and electrotaxis, the process by which cells detect an electric field and orient their migration along its direction, has emerged as a potential cornerstone of the endogenous wound healing response. Despite recognition of the importance of electrotaxis in wound healing, no experimental system to date demonstrates that the actual closing of a wound can be accelerated solely by the electrotaxis response itself, and in vivo systems are too complex to resolve cell migration from other healing stages such as proliferation and inflammation. This uncertainty has led to a lack of standardization between stimulation methods, model systems, and electrode technology required for device development. In this paper, we present a 'healing-on-chip' approach that is a standardized, low-cost, model for investigating electrically accelerated wound healing. Our device provides the first convergent field geometry used in a stimulation device. We validate this device by using electrical stimulation to close a $1.5 \mathrm{~mm}$ gap between two large $\left(30 \mathrm{~mm}^{2}\right)$ primary skin keratinocyte layers to double the rate of healing over an unstimulated tissue. This proves that convergent electrotaxis is both possible and can accelerate healing, and offers a new 'healing-on-a-chip' platform to explore future bioelectric interfaces.
\end{abstract}

\section{Introduction}

Since du Bois-Reymond first characterized the naturally occurring 'wound current' nearly two centuries ago (1), there has been significant interest in applying external electrical stimulation to improve wound healing (2-4). The potential for this approach is becoming increasingly apparentfor instance, numerous, recent, in vivo studies show some improvement in skin healing in animal models upon electric field stimulation (5-10), while in vitro assays have demonstrated control of cells and simple tissues using spatially programmed electric cues (11-13). Further, given the increasing prevalence and healthcare burden of wound treatments $(14,15)$, new technologies to expedite and improve wound care are sorely needed. However, despite these and other studies over the past several decades, the few extant commercial products have demonstrated mixed results (16-19), and bioelectric wound therapy is far from the standard of care. This discrepancy is due to broad gaps in both technology development and biological knowledge describing how electrical stimulation may act to improve wound healing. Technologically, optimum stimulation parameters 
37 for field strength, biointerface design, and current delivery mode remain unclear $(20,21)$.

38 Biologically, there is uncertainty about how the key wound healing mechanisms--cell migration,

39 proliferation, and inflammation — are affected by electric stimulation (2). This uncertainty has

40 resulted in a lack of standardization in stimulation schemes, model systems, and technology that

41 can all lead to issues of reproducibility and long design iterations time that have slowed progress

42 (22-24).

44 Here, we begin to address this problem by integrating a popular technical approach used in other 45 branches of biotechnology — 'organ-on-a-chip' systems - to reduce the complexity of biomedical 46 problems to something both tractable and eventually translatable. Organ-on-a-chip (OoC) 47 platforms are in vitro model systems that capture a specific and critical physiological behavior of 48 the in vivo system in a standardized, rapid, lower-cost in vitro model. To date, OoCs have clearly 49 proven their value in other fields by aiding discoveries and treatments for lung, gut, and vascular 50 pathologies (25-27). Here, we use an OoC approach to integrate a 'healing-on-a-chip' platform 51 with a custom electrobioreactor designed from the ground up to investigate electrically accelerated 52 wound healing.

54 While there are many effects that applied electrical stimulation may have on tissue growth and 55 healing, the best-characterized is electrotaxis - the directed motion of cells in response to an 56 electric current. Electrotaxis is seen in over 20 cell types across multiple organisms where cells 57 sense and track electrochemical potential gradients $(\sim 1 \mathrm{~V} / \mathrm{cm})$ that emerge during development 58 and injury healing (28-30). The mechanism of detection is thought to be electrophoresis of charged 59 membrane-bound receptors in the presence of an electric field, resulting in an asymmetric 60 distribution of these proteins that triggers downstream signaling of the cell migration machinery 61 (31). In vivo, these fields result in the center of a skin wound being negatively polarized relative 62 to the periphery of the wound (32,33). Direct current fields are analogous to fields in vivo (34), in 63 contrast to the pulsed DC or AC stimulation used in many in vivo studies (5), and are sufficient to 64 induce the electrotaxis response. However, electrotaxis has primarily been studied in isolated 65 single cells to elucidate the molecular biology of the process, and at present there is no study, either 66 in vitro or in vivo that conclusively indicates that electrotaxis itself can accelerate wound closure.

67 This gap stems from the technological limitations of current devices used to study electrotaxis. 
68 Nearly all devices use a single electrode pair to apply a uniform, unidirectional field across

69 tissues - such a field would cause one side of a wound to close and the other side to worsen. In

70 vivo, the wound field converges on the center of a wound, so a new device design is required to

71 capture this characteristic. In addition to this stimulation limitation, most studies and devices do

72 not generalize well to macroscale tissues and wounds since precise tissues with reproducible,

73 millimetric wounds must be grown inside the electrobioreactor. Finally, macroscale cell migration

74 requires stable electrical stimulation over many hours, and the common, bleach-based electrode

75 preparation process is insufficient for long-term stimulation ( $>4$ hours). An ideal bioelectric

76 'wound-on-a-chip' platform should address these issues.

78 Here we build on our prior work $(11,12)$ to create a new electrobioreactor to study healing in a

79 macroscale skin-on-a-chip model using primary mouse skin monolayers which migrate toward

80 the cathode when stimulated (Fig. 1), and use electrical stimulation to accelerate closure of 1.5

$81 \mathrm{~mm}$ large model skin wounds by at least $2 \mathrm{X}$ over unstimulated skin layers (Figs. 2, 3). To

82 accomplish this, we developed new electrotaxis infrastructure specifically designed for the

83 constraints of wound healing, delivering a sustained converging electric field to a tissue (Fig. 1).

84 With this device, we were able to engineer and stimulate the largest tissues yet tested with

85 electrotaxis $\left(30 \mathrm{~mm}^{2}\right)$ for 12 hours, while also exploring the consequences of overstimulation.

\section{Results}

88 Our new electrobioreactor significantly departs from extant electrotaxis systems by generating an

89 electric field that converges at the center of model wounds, and functions as follows. The device

90 consists of an acrylic insert clamped to a standard tissue culture dish, holding electrodes and

91 agarose salt bridges in position (Fig. 1A, see ESI Methods for fabrication details). Three

92 chloridized silver stimulation electrodes (anodes at left and right, cathode at center) are isolated

93 from each other in separate saline reservoirs and electrical contact with the culture media is

94 provided by $4 \%$ agarose w/v salt bridges cast inside the insert, one per reservoir (Fig. 1B). The

$95 \sim 500 \mu \mathrm{m}$ thin, laser-milled, agarose bridge serves as a central cathode and is aligned directly

96 over the wound site (Fig. 1C, see '*'). The result is a stable, uniform field that converges upon

97 the central electrode as confirmed by simulation (Fig. 1D,E). To reliably generate reproducible

98 tissues and linear wounds, we use a silicone stencil templating method $(12,35)$ to prepare 
99 confluent monolayers the evening before an experiment. We then assemble the electrobioreactor

100 over these tissues prior to imaging. For these experiments, we use layers of keratinocytes from

101 primary skin cultured under basal conditions optimized for electrotaxis (11). After the tissues

102 have grown, the stencils are removed, and the device is clamped over the cells, aligned such that

103 that the central slit electrode is in the gap between cells (Fig. 1E). Then, a computer-controlled

104 source meter (Keithley 2450) is connected to each pair of electrodes (left-center and right-

105 center), with both sources sharing the central cathode, to supply an electric current. The field

106 within the chamber is continuously monitored by a digital oscilloscope and the current output of

107 each source meter is adjusted via closed-loop control to maintain a constant $2 \mathrm{~V} / \mathrm{cm}$ field

108 strength directed toward the central cathode. We specifically chose this field strength as it has

109 previously been validated and was chosen to amplify the approximate field strength experienced

110 in vivo (28). To extend cathode lifetime, only one source was active at a time, alternating

111 between left-center and right-center stimulation every 30 seconds. Oxygen delivery and waste

112 management are handled by perfusing fresh media through the bioreactor at $2 \mathrm{~mL} / \mathrm{h}$, turning over

113 the chamber volume $\sim 11$ times per hour. The resulting system provides a robust convergent field

114 to viable cells.

116 Stable DC stimulation and cell viability require that the electrodes remain intact throughout an

117 entire experiment, so optimization of electrode chemistry is an important consideration. Virtually

118 all DC electrotaxis chambers use an anode and a cathode to inject Faradaic current through a

119 sample, using combinations of salt bridges, media perfusion, and heavy buffering to prevent the

120 buildup of toxic electrochemical byproducts or harmful $\mathrm{pH}$ changes due to electrolysis at the

121 electrodes $(20,36,37)$. Because the current used in our device is moderate ( 6-10 mA) and the

$1221.5 \mathrm{~mm}$ gap between tissues is relatively large, the central cathode must be able to sink current

123 for an extended period to induce tissue convergence, ideally 12 hours or more. To support this,

124 our system uses electrically chloridized silver foil as electrodes, which degrades at the cathode

125 into ionic silver and chloride during stimulation. This reaction is more favorable than the 

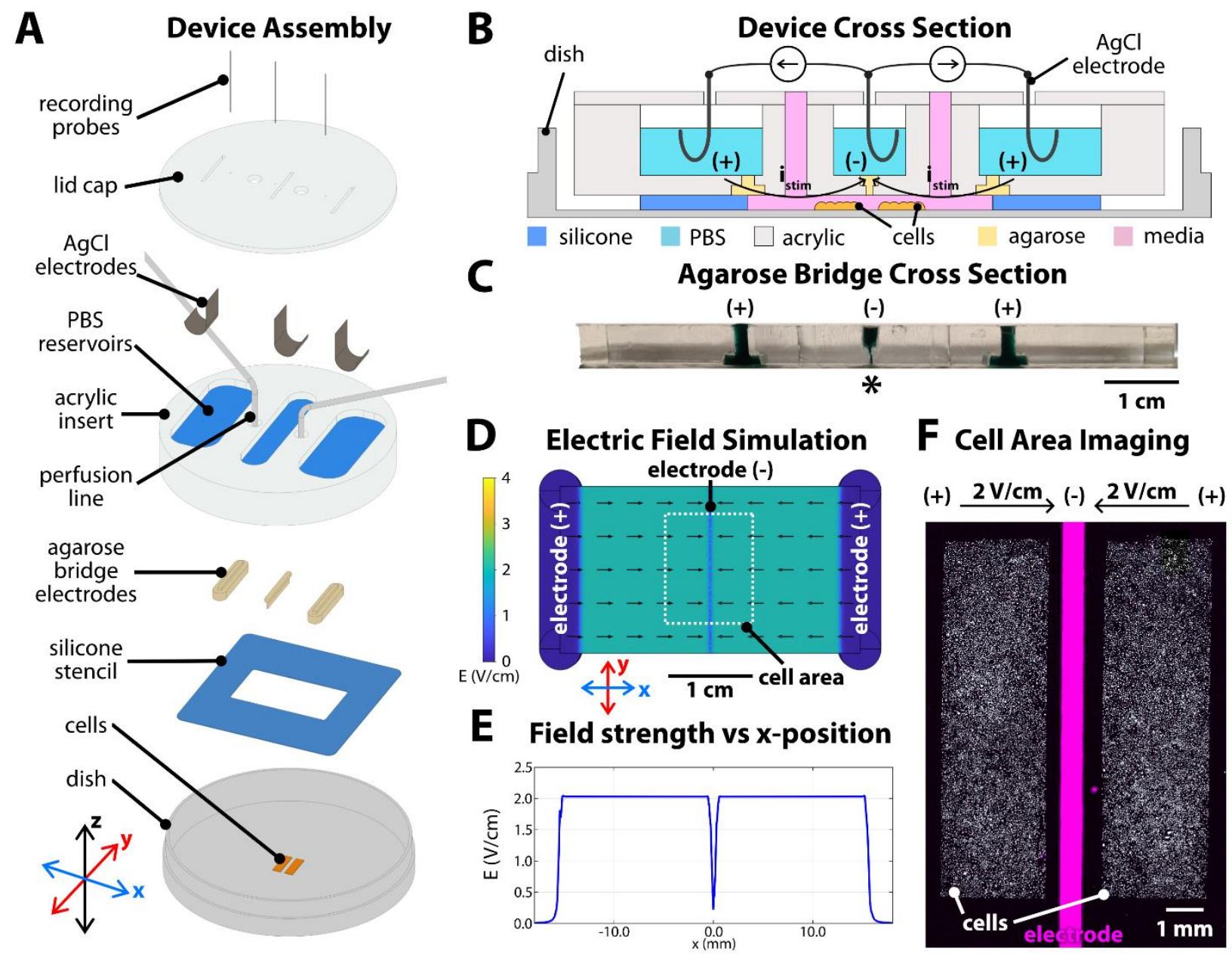

Fig. 1: Convergent field stimulation device. (A) Layer-based assembly of the bioreactor onto a tissue culture dish. Cells are patterned in the center of the dish, then a $250 \mu \mathrm{m}$-thick silicone stencil is placed to define the stimulation area and height. Agarose bridges are cast inside an acrylic insert, then clamped into the dish and against the silicone stencil. The reservoirs on the topside of the acrylic insert are filled with phosphate-buffered saline (PBS).

Chloridized silver electrodes and titanium wire recording probes are inserted in each reservoir, all held in place by a lid cap. (B) Device cross-section sketch and (C) photograph of the sectioned agarose bridges stained with green food coloring for contrast. The narrow cathode is labeled with '*'. (D) Numeric simulation of the electric field in the device, showing constant $2 \mathrm{~V} / \mathrm{cm}$ field strength converging toward the center, with a steep drop-off in strength starting $\pm 500 \mu \mathrm{m}$ from the center. (E) Simulated field strength versus x-position in the device. (F) Microscope capture of the central area of the assembled device, showing the central electrode $500 \mu \mathrm{m}$ wide positioned between the two tissues. The cells (white) were labeled with a Cy5 lipophilic dye and the outline of the central electrode was visualized with a DAPI filter set $\left(\lambda_{\text {ex }} / \lambda_{\text {em }} 358 / 461 \mathrm{~nm}\right)$ and filled via post-processing in ImageJ (magenta).

hydrolysis cathodal half-reaction, which evolves hydrogen gas from the solution and increases

$127 \mathrm{pH}$ (37). This allows for safe stimulation until $\mathrm{AgCl}$ is depleted at the cathode, when evolution of

$128 \mathrm{H}_{2}$ then becomes favorable and $\mathrm{pH}$ increases rapidly, which can cause cytotoxicity. Therefore,

129 sufficient chloridization of the silver foil is paramount for extended electrode lifetime. We

130 compared our chloridization method with bleach immersion, another technique commonly used

131 to chloridize silver. We performed repeated cyclic voltammetry to compare electrode 
132 preparations and found that our method of electroplating silver chloride resulted in more stable

133 cathodes (see ESI, Fig. S1). Our combined approach of robust silver chloridization, agarose

134 diffusion barriers to prevent ionic silver reaching the tissue, and media perfusion integrates

135 numerous best practices to maximize cell viability during stimulation in our device, allowing for

136 extended wound healing experiments.

138 To evaluate this platform for in vitro healing, we patterned two 10 × $3 \mathrm{~mm}$ tissues spaced $1.5 \mathrm{~mm}$ 139 apart with the central cathode aligned over the wound center (Fig. 1F). The acrylic outline of the 140 central cathode slit fluoresces weakly when imaged using a standard DAPI filter set, so the 141 alignment between the central cathode and the tissues could be tuned and verified. We then 142 applied convergent electrical stimulation over 12 hours following a 30-minute control period 143 without field, with striking results (Fig. 2, Video S1). In the non-stimulated control case, cell 144 proliferation and migration lead to the slow expansion of tissues and gradual, but incomplete 145 closure of the wound over 12 hours ( $50 \%$ closure, $\mathrm{N}=3)$. However, convergent bioelectric

146 stimulation led to complete closure between 11-12 h $(\mathrm{N}=3)$. More specifically, the edge

147 migration speed was twice as fast in the stimulated case as in the control, measuring $29.4 \pm 3.3$

$148 \mu \mathrm{m} / \mathrm{h}$ and $62.2 \pm 8.1 \mu \mathrm{m} / \mathrm{h}$ for the control and stimulated cases, respectively. To conclusively

149 attribute this effect to electrical stimulation rather than temperature effects (Joule heating has

150 been linked to increased migration speeds in prior studies $(31,38)$ ) we monitored the device 151 temperature during stimulation (Fig. S2). The steady state temperature rose from $37{ }^{\circ} \mathrm{C}$ to $38^{\circ} \mathrm{C}$, 152 and this $3 \%$ increase is unlikely to account for the $100+\%$ increase in migration speed during 153 stimulation. We hypothesize that perfusion and media turn over helps to exchange heat and 154 mitigate any effects from Joule heating. Taken together, this is the first demonstration of 155 convergent field stimulation accelerating in vitro wound healing, and the results prove that 156 electrotaxis alone is sufficient for this closure. 

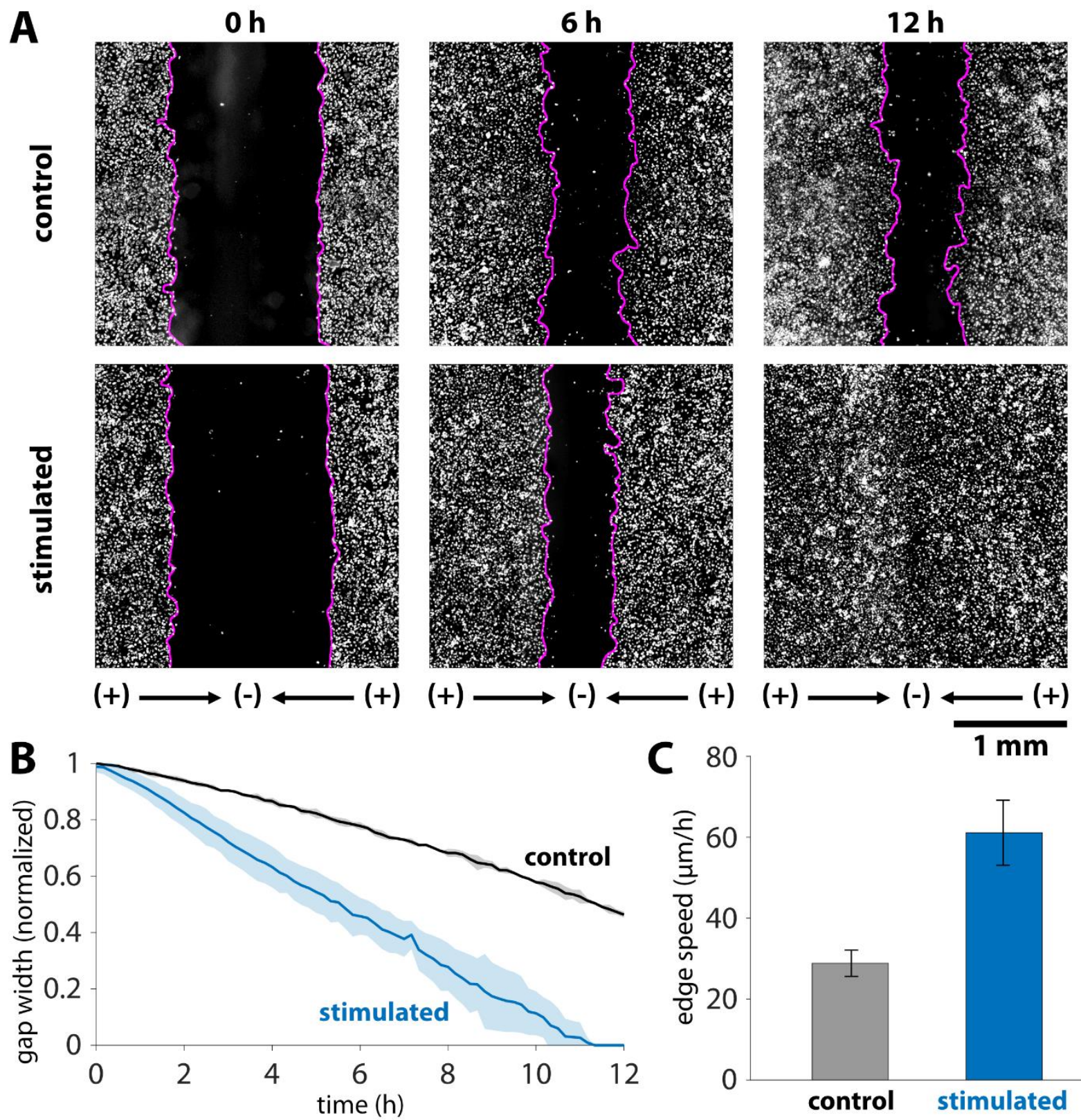

Fig. 2. Dynamics of accelerated wound closure of keratinocyte monolayers. (A) Timepoint comparison of stimulation versus control for keratinocytes labeled with a Cy5 cytoplasmic dye. Gap boundaries are demarked by magenta lines. Initial gap between tissues was $1.5 \mathrm{~mm}$, and this gap closed by 12 hours in the stimulated case, while roughly $50 \%$ of the gap remained in the control case. (B) Gap closure normalized to the initial gap width for $\mathrm{N}=3$ tissues in each condition. Shaded region represents standard deviation. (C) Edge expansion speeds averaged over an $8 \mathrm{~h}$ period. The average edge expansion was $29.4 \pm 3.3 \mu \mathrm{m} / \mathrm{h}$ and $62.24 \pm 8.1 \mu \mathrm{m} / \mathrm{h}$ for the control and stimulated cases, respectively. $\mathrm{N}=6$ edges in each case and error bars represent standard deviation.

157 To better characterize device performance and its effects on large scale tissue growth and 158 motion, we performed particle image velocimetry (PIV) on each tissue. Representative

159 horizontal velocity kymographs for both the control and convergent stimulation cases are shown 
160 in Fig. 3 (compare with Video S1). To provide context of spatial dynamics within a given tissue,

161 we show representative heatmaps of horizontal velocity and line integral convolution (LIC)

162 migration maps to visualize the overall flow of cellular motion at 4 hours after the onset of

163 stimulation (steady state). Throughout the control tissue (Figs. 3A-C), there is little net outwards

164 motion, except for slow expansion at the edges. Disorder is apparent in the velocity and

165 migration maps of the control tissues, which lack large regions of coordinated movement, as

166 expected for non-stimulated tissues (Figs. 3B,C). In contrast, bioelectric stimulation resulted in

167 nearly uniformly high-speed motion throughout the tissue, converging on the gap within 15

168 minutes of the field turning on, as visualized in the velocity and migration maps (Figs. 3D-F).

169 The large number of parallel streaklines along the stimulation direction in the migration map

170 demonstrates highly coordinated motion across the tissue in alignment with the stimulus (Figs.

$1713 \mathrm{~F})$. These visualizations reveal that the electric field acts a global migration cue across a large

172 area, confirming that cells experience a highly uniform field as predicted by simulation (Fig.

173 1D,E).

174

175 Having demonstrated that the in vitro healing process can be electrically accelerated overall, we

176 next characterized cellular responses specifically during the final stages of wound closure.

177 Unlike traditional electrotaxis chambers where the electrodes are significantly distal to the tissue

178 to ensure a uniform field, our healing-on-a-chip device requires a central electrode to focus cell

179 migration into the wound zone. Since the central electrode has a finite width $(\sim 500 \mu \mathrm{m}$ here $)$ that

180 is smaller than the wound, this means that tissues will eventually pass underneath the electrode

181 and enter the 'electrode shadow' during the final stages of healing and convergence. Any

182 discrete electrode produces field non-uniformities close to its surface, so as cells enter the

183 electrode shadow, they will experience a very different field than out in the fully developed

184 zones far from the center. Our simulation predicts a sharp decrease in electric field strength that

185 begins about $500 \mu \mathrm{m}$ on either side of the central cathode above the convergence region (Fig.

186 4A). We quantified the actual effects of the central field singularity by stimulating closed tissues

187 for 6 hours and using a live nuclear dye to track cells in that central zone (Video S2). We

188 averaged PIV across the region surrounding the closure zone over the stimulation period (Fig.

189 4B, asterisks and error bars) and fit a sigmoid function to the data (Fig. 4B, inset) showing that

190 there is a strong, steady-state response far from the central electrode that steadily weakens as 

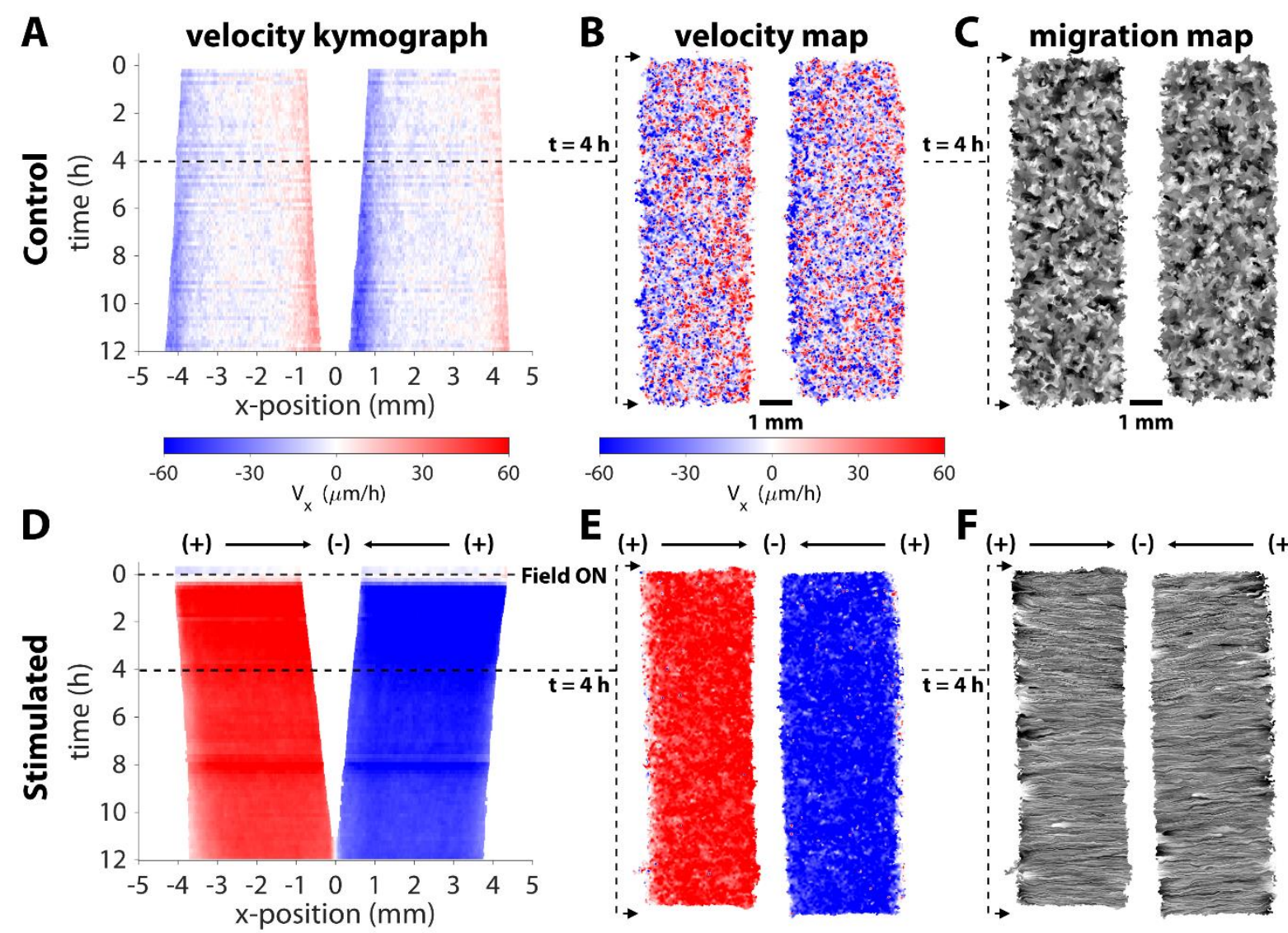

$\mathbf{E}_{(+)} \longrightarrow(-) \longleftarrow(+)$
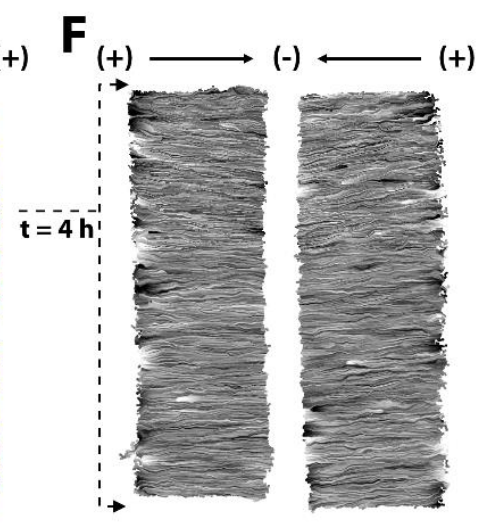

Fig. 3. Convergent stimulation results in coherent migration response towards the center. Representative kymograph of Vx averaged across x-position in a control (A) and stimulated (D) tissue pair. The control tissues expand uniformly outwards, while the stimulated tissues converge towards the cathode with uniformly high speed across the tissue area $(\sim 60 \mu \mathrm{m} / \mathrm{h}$ directed towards the gap). Horizontal velocity maps (B,E) and migration maps $(\mathrm{C}, \mathrm{F})$ in representative tissue pairs.

191 cells approach the central electrode and enter the electrode shadow (Fig. 4, dashed blue line;

192 magenta zone shows electrode shadow). While this local weakening of the electrotactic response

193 closely resembled the trend in our simulations, cells nonetheless continued to directionally

194 migrate deep into the electrode shadow zone, only to dropping to $<50 \%$ of the steady state

195 velocity once cells were $\sim 100 \mu \mathrm{m}$ off the electrode midline. These data show that the effective

196 electrode size is smaller than its physical, $500 \mu \mathrm{m}$ width (Fig. 4B, compare dotted black

197 boundaries to electrode boundaries). This means that even relatively large electrodes can still

198 promote last-mile healing.

200 Critically, we also observed potential consequences to continued electrical stimulation after a

201 wound had closed. As has been noted previously (39), electrotaxis appears to override basic

202 cellular safety mechanisms, such as contact inhibition, meaning that cells will continue trying to 
203 directionally migrate as long as stimulation is active. In our wound healing model, this meant

204 that stimulating after a tissue had closed would continue to drive cell migration towards where

205 the center of the wound had previously been. This inevitably caused an increase in local cell

206 density, and we measured a $>2 \mathrm{X}$ increase (from 750 to 1600 cells $/ \mathrm{mm}^{2}$ ) in cell density under the

207 electrode shadow relative to density distal to the central electrode (Fig. 4C-E), showing there is

208 potential for over-densification of cells driven by post-healing stimulation. Comparing individual

209 cell trajectories within the central zone confirmed that cells within the electrode shadow

210 translated horizontally a lower distance than those that were farther away (Fig. 4F,G). This

211 reduction in overall translation extended, in a graded fashion, outwards $500 \mu \mathrm{m}$ from the center

212 in either direction, consistent with the reduction of speed cells experience as they enter the

213 electrode shadow. Nevertheless, there is net migration towards the center, even for cells that

214 were initially positioned under the electrode, suggesting again that the electrode's influence

215 extends underneath its width despite significant weakening of the effective field strength.

217 We suggest two reasons that the 'effective electrode' size would be smaller than its physical size.

218 First, the threshold field strength that elicits an electrotaxis response is lower than the $2 \mathrm{~V} / \mathrm{cm}$ we

219 target in stimulation. As the field strength rolls off, it is still 'therapeutic' for some time, given

220 that physiological field strengths are on the order of $1 \mathrm{~V} / \mathrm{cm}$ (28). Second, the monolayers carry

221 some memory of the electrotaxis response that continues to influence their responses after the

222 stimulus changes (11). Keratinocytes polarize in response to the field stimulus, and this

223 polarization takes time to decay once a stimulus is no longer detected. This could lead to cells

224 effectively coasting, unguided, during the last gap before tissue closure. 

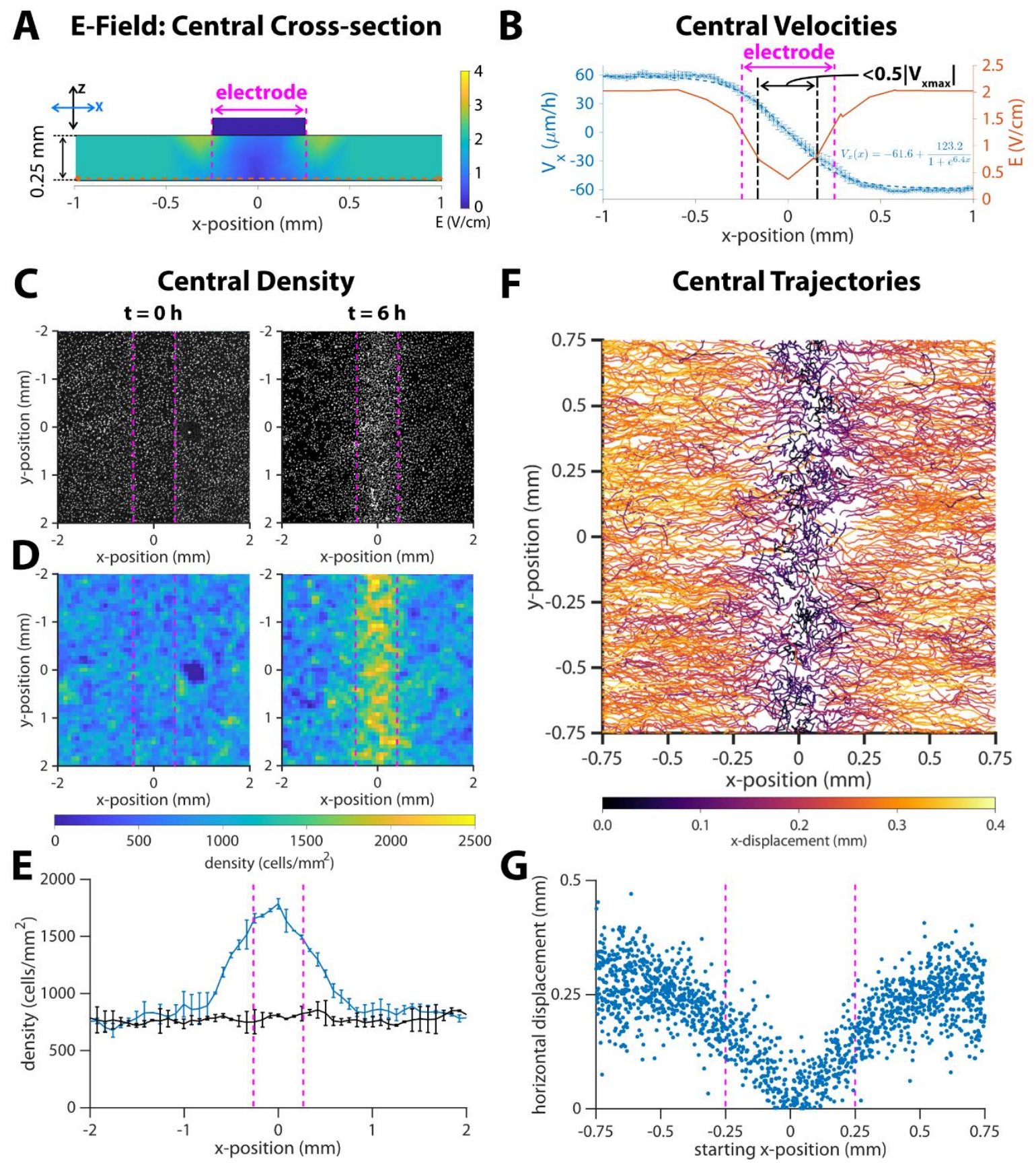

Fig. 4. Response of migrating cells near the center of a closed tissue. Dotted vertical magenta lines indicate approximate cathode boundaries in each respective graph. (A) Numeric simulation results for the electric field of the channel in cross section. The horizontal dotted line is the section of the electric field plotted in the next panel. (B) Average horizontal velocity plotted as blue *'s with error bars representing standard deviation $(\mathrm{N}=2)$. The dashed blue line indicates a least-squares fit of a sigmoid function to the data, and the formula for this fit is inset in the lower right quadrant of the plot. The region where the horizontal velocity's magnitude drops to $<50 \%$ of the steady state value is marked by dot-dash black lines. Predicted strength from numeric simulation (reproduced from Fig. 1E) is plotted as a red solid line. (C) DAPI images of cell nuclei and (D) density maps for tissues at the onset of stimulation $(\mathrm{t}=0 \mathrm{~h})$ and after six hours of stimulation $(\mathrm{t}=6 \mathrm{~h})$. (E) Average cell density versus $\mathrm{x}$-position, where $\mathrm{x}$ $=0$ is the center of the cathode. Error bars represent standard deviation $(\mathrm{N}=2)$. (F) Montage of 6-hour trajectories of individual cells in proximity with the center. Each track is colored by net $\mathrm{x}$-displacement. (G) Total horizontal displacement of cell versus its starting $\mathrm{x}$-position relative to the center of the cathode at $\mathrm{x}=0$. 


\section{Discussion \& Conclusion}

226 Overall, we present a bioelectric, healing-on-a-chip (HoC) platform designed specifically to

227 study the role of electrotaxis and other electrical phenomena in wound healing. Unique for 228 electrical stimulation bioreactors, our approach creates a field stimulation pattern that mimics

229 that found in wounds in vivo, with the field converging at the center of the wound gap. This

230 capability allows us to directly explore the actual healing process, rather than purely uni-

231 directional cell migration. Therefore, our platform allows study of the in vitro healing process

232 spanning initial injury, 'first contact' as the sides of the wound meet and, critically, post-closure

233 behavior after the wound has healed. Using this platform and unoptimized stimulation

234 parameters, we demonstrate $\sim 2 \mathrm{X}$ acceleration of wound closure in an in vitro skin layer model

235 due solely to electrotactic effects. To our knowledge, this is the first demonstration and

236 visualization of electrotaxis itself accelerating a healing process. The stability, reproducibility,

237 and programmability of the platform make it suitable to deeply explore key technological and

238 biological questions, and we have taken care to ensure the device is easily replicable and

239 accessible to a broad audience.

241 That even naïve stimulation had a strong, positive effect on in vitro healing is encouraging, and

242 establishes a clear baseline against which future parameter optimization studies can be

243 compared. This approach could be critical to the field as standardization and optimization of

244 stimulation approaches remains an open question. To hint at this, we explored the effects of

245 stimulating beyond initial closure of the wound by electrically stimulating a closed tissue, and

246 the resulting cellular pile-up indicates both the potency of electrotaxis to drive migration and the

247 importance of being able to fine-tune and intelligently adjust stimulation in practice to avoid

248 detrimental effects of overstimulation. Such cellular pile-ups also speak more fundamentally to

249 the role of electrotaxis as a tool to modulate and explore interactions at the boundaries between

250 tissues.

252 While we specifically investigated healing in monolayers of primary skin cells here, wound

253 healing in vivo clearly involves complex coordination across multiple cell types (e.g.

254 macrophages and immune cells, fibroblasts, and vascular cells, and epidermal cells) and phases, 255 (e.g inflammation, granulation, and re-epithelialization) (2). That our platform supports pre- 
engineering tissue configurations means that co-cultures or more complex tissue models can be

257 grown first and then incorporated into the bioreactor to allow more complex studies on healing.

258 When linked to stimulation optimization approaches, it may be possible to determine modalities

259 that preferentially target a given cell type, or process such as proliferation vs. migration during

260 healing. Again, these questions benefit from a field geometry that enables a healing phenotype.

262 Finally, our bioelectric 'Healing-on-a-Chip' approach is fully open and intended to be modified 263 and tailored for a variety of applications. We provide complete design files, computational

264 models, and stimulation code (Provided via a GitHub repository:

265 https://github.com/CohenLabPrinceton/SCHEEPDOG), and the basic approach lends itself to

266 easy customization. For instance, electrode shape, size, number, and location can easily be

267 adjusted without additional cost or significant complexity. Field stimulation strategies can be

268 tested by attaching any desired power supplies or running arbitrary stimulation code to activate

269 electrode sequences. Our autofluorescence alignment approach makes it possible to accurately

270 align a given electrode configuration to a given wound and removes much of the ambiguity and

271 difficulty this process would normally introduce. We hope the demonstrations here and

272 flexibility of the device can help accelerate healing-on-a-chip research, improve translation for

273 future in vivo applications, and even support new, research on general interactions between

274 colliding tissues.

275

276 Acknowledgements

277 We gratefully acknowledge Prof. Danelle Devenport and Katie Little at Princeton University for 278 providing primary keratinocytes and culture support. Research reported in this publication was

279 supported by the National Center for Advancing Translational Sciences (NCATS), a component

280 of the National Institute of Health (NIH) under award number TL1TR003019 (TJZ). Further

281 support was provided by National Institutes of Health grant R35GM13357401 (DJC, GS). The

282 content is solely the responsibility of the authors and does not necessarily represent the official

283 views of the National Institutes of Health. 


\section{References}

1. Bois-Reymond E du. Untersuchungen uber thierische Elektricitat. Berlin, Reimer. 1848;1.

2. Tai G, Tai M, Zhao M. Electrically stimulated cell migration and its contribution to wound healing. Burns \& trauma. Narnia; 2018;6(1).

3. Anderson CA, Hare MA, Perdrizet GA. Wound healing devices brief vignettes. Advances in wound care. Mary Ann Liebert, Inc. 140 Huguenot Street, 3rd Floor New Rochelle, NY 10801 USA; 2016;5(4):185-190.

4. Hunckler J, De Mel A. A current affair: electrotherapy in wound healing. Journal of multidisciplinary healthcare. Dove Press; 2017;10:179.

5. Long Y, Wei H, Li J, Yao G, Yu B, Ni D, et al. Effective Wound Healing Enabled by Discrete Alternative Electric Fields from Wearable Nanogenerators. ACS Nano. 2018;12(12):12533-12540.

6. Liang Y, Tian H, Liu J, Lv YL, Wang Y, Zhang JP, et al. Application of stable continuous external electric field promotes wound healing in pig wound model. Bioelectrochemistry [Internet]. Elsevier LTD; 2020;135:107578. Available from: https://doi.org/10.1016/j.bioelechem.2020.107578

7. Jang HK, Oh JY, Jeong GJ, Lee TJ, Im GB, Lee JR, et al. A disposable photovoltaic patch controlling cellular microenvironment for wound healing. International Journal of Molecular Sciences. 2018;19(10).

8. Kai H, Yamauchi T, Ogawa Y, Tsubota A, Magome T, Miyake T, et al. Accelerated Wound Healing on Skin by Electrical Stimulation with a Bioelectric Plaster. Advanced Healthcare Materials. 2017;6(22):1-5.

9. Yu C, Xu ZX, Hao YH, Gao YB, Yao BW, Zhang J, et al. A novel microcurrent dressing for wound healing in a rat skin defect model. Military Medical Research [Internet]. Military Medical Research; 2019;6(1):1-9. Available from: https://mmrjournal.biomedcentral.com/track/pdf/10.1186/s40779-019-0213-x

10. Wang X-F, Li M-L, Fang Q-Q, Zhao W-Y, Lou D, Hu Y-Y, et al. Flexible electrical stimulation device with Chitosan-Vaseline ${ }^{\circledR}$ dressing accelerates wound healing in diabetes. Bioactive materials. Elsevier; 2020;6(1):230-243.

11. Zajdel TJ, Shim G, Wang L, Rossello-Martinez A, Cohen DJ. SCHEEPDOG: Programming Electric Cues to Dynamically Herd Large-Scale Cell Migration. Cell Systems [Internet]. Elsevier Inc.; 2020;10(6):506-514.e3. Available from: https://doi.org/10.1016/j.cels.2020.05.009

12. Cohen DJ, Nelson WJ, Maharbiz MM. Galvanotactic control of collective cell migration in epithelial monolayers. Nature materials. Nature Publishing Group; 2014;13(4):409-417.

13. Gokoffski KK, Jia X, Shvarts D, Xia G, Zhao M. Physiologic electrical fields direct retinal ganglion cell axon growth in vitro. Investigative ophthalmology \& visual science. The Association for Research in Vision and Ophthalmology; 2019;60(10):3659-3668.

14. Padula WV, Delarmente BA. The national cost of hospital-acquired pressure injuries in the United States. International Wound Journal. 2019;16(3):634-640.

15. Martinengo L, Olsson M, Bajpai R, Soljak M, Upton Z, Schmidtchen A, et al. Prevalence of chronic wounds in the general population: systematic review and meta-analysis of observational studies. Annals of epidemiology. Elsevier; 2019;29:8-15.

16. Morris C. Bio-electrical stimulation therapy using POSiFECT®RD. Wounds UK. 2006;2(4):112-116. 
17. Kloth LC. Electrical Stimulation Technologies for Wound Healing. Advances in Wound Care. 2014;3(2):81-90.

18. Kim H, Izadjoo M. Antibiofilm efficacy evaluation of a bioelectric dressing in mono-and multi-species biofilms. Journal of wound care. MA Healthcare London; 2015;24(Sup2):S10-S14.

19. Kim H, Park S, Housler G, Marcel V, Cross S, Izadjoo M. An overview of the efficacy of a next generation electroceutical wound care device. Military Medicine. Oxford University Press; 2016;181(suppl_5):184-190.

20. Zhao Z, Zhu K, Li Y, Zhu Z, Pan L, Pan T, et al. Optimization of Electrical Stimulation for Safe and Effective Guidance of Human Cells. Bioelectricity. Mary Ann Liebert, Inc., publishers 140 Huguenot Street, 3rd Floor New ...; 2020;

21. Khouri C, Kotzki S, Roustit M, Blaise S, Gueyffier F, Cracowski J-L. Hierarchical evaluation of electrical stimulation protocols for chronic wound healing: An effect size meta-analysis. Wound Repair and Regeneration. Wiley Online Library; 2017;25(5):883891.

22. Isseroff RR, Dahle SE. Electrical stimulation therapy and wound healing: where are we now? Advances in wound care. Mary Ann Liebert, Inc. 140 Huguenot Street, 3rd Floor New Rochelle, NY 10801 USA; 2012;1(6):238-243.

23. Kloth LC. Electrical stimulation for wound healing: a review of evidence from in vitro studies, animal experiments, and clinical trials. The international journal of lower extremity wounds. Sage Publications Sage CA: Thousand Oaks, CA; 2005;4(1):23-44.

24. Ashrafi M, Alonso-Rasgado T, Baguneid M, Bayat A. The efficacy of electrical stimulation in lower extremity cutaneous wound healing: a systematic review. Experimental dermatology. Wiley Online Library; 2017;26(2):171-178.

25. Zhang B, Korolj A, Lai BFL, Radisic M. Advances in organ-on-a-chip engineering. Nature Reviews Materials. Nature Publishing Group; 2018;3(8):257-278.

26. Wang Z, Samanipour R, Koo K, Kim K. Organ-on-a-chip platforms for drug delivery and cell characterization: A review. Sens. Mater. 2015;27(6):487-506.

27. Zhang B, Radisic M. Organ-on-a-chip devices advance to market. Lab on a Chip. Royal Society of Chemistry; 2017;17(14):2395-2420.

28. McCaig CD, Song B, Rajnicek AM. Electrical dimensions in cell science. Journal of cell science. The Company of Biologists Ltd; 2009;122(23):4267-4276.

29. Cortese B, Palama IE, D'Amone S, Gigli G. Influence of electrotaxis on cell behaviour. Integrative Biology. Oxford University Press; 2014;6(9):817-830.

30. Kennard AS, Theriot JA. Osmolarity-independent electrical cues guide rapid response to injury in zebrafish epidermis. Elife. eLife Sciences Publications Limited; 2020;9:e62386.

31. Allen GM, Mogilner A, Theriot JA. Electrophoresis of cellular membrane components creates the directional cue guiding keratocyte galvanotaxis. Current Biology. Elsevier; 2013;23(7):560-568.

32. Wahlsten O, Skiba JB, Makin IRS, Apell SP. Electrical field landscape of two electroceuticals. Journal of Electrical Bioimpedance. 2016;7(1):13-19.

33. Shen Y, Pfluger T, Ferreira F, Liang J, Navedo MF, Zeng Q, et al. Diabetic cornea wounds produce significantly weaker electric signals that may contribute to impaired healing. Scientific Reports [Internet]. 2016;6(June):1-12. Available from: https://www.nature.com/articles/srep26525.pdf 
374 34. McCaig CD, Rajnicek AM, Song B, Zhao M. Controlling cell behavior electrically: current views and future potential. Physiological reviews. American Physiological Society; 2005;

35. Heinrich MA, Alert R, LaChance JM, Zajdel TJ, Košmrlj A, Cohen DJ. Size-dependent patterns of cell proliferation and migration in freely-expanding epithelia. Rosenblatt $\mathrm{J}$, Stainier DY, Kabla A, editors. eLife [Internet]. eLife Sciences Publications, Ltd; 2020 Aug;9:e58945. Available from: https://doi.org/10.7554/eLife.58945

36. Schopf A, Boehler C, Asplund M. Analytical methods to determine electrochemical factors in electrotaxis setups and their implications for experimental design. Bioelectrochemistry. Elsevier; 2016;109:41-48.

37. Li M, Wang X, Rajagopalan P, Zhang L, Zhan S, Huang S, et al. Toward Controlled Electrical Stimulation for Wound Healing Based on a Precision Layered Skin Model. ACS Applied Bio Materials. ACS Publications; 2020;

38. Ream RA, Theriot JA, Somero GN. Influences of thermal acclimation and acute temperature change on the motility of epithelial wound-healing cells (keratocytes) of tropical, temperate and Antarctic fish. Journal of experimental biology. The Company of Biologists Ltd; 2003;206(24):4539-4551.

39. Zhao M. Electrical fields in wound healing — an overriding signal that directs cell migration. Seminars in cell \& developmental biology. Elsevier; 2009. p. 674-682. 